Jermy Rifkin, aber auch um Vertreter von Institutionen wie Bischof Reinhard Marx oder Hubert Weiger vom BUND sowie um Unternehmer wie Johannes Rahe, Huschmand Sabet und Michael Otto.

2) Mercosur, spanisch für Mercado Común del Sur („Gemeinsamer Markt des Südens“), bzw. Mercosul, portugiesisch für Mercado Comum do Sul, ist ein Binnenmarkt mit einem Bruttoinlandsprodukt von etwa 1 Billion US-Dollar. Mitglieder sind Argentinien, Brasilien, Paraguay, Uruguay und Venezuela, assoziierte Mitglieder Bolivien, Chile, Ecuador, Kolumbien und Peru.

$《 \gg$

\section{Das „Banse-Rhizom“ Aktuelle Einblicke in Technologie und Philosophie}

\author{
H.-J. Petsche, M. Bartiková, A. Kiepas \\ (Hg.): Erdacht, gemacht und in die Welt \\ gestellt: Technik-Konzeptionen zwischen \\ Risiko und Utopie. Festschrift für Gerhard \\ Banse. Berlin: trafo verlag, 2006, 419 S., \\ ISBN 3-89626-612-8, Euro 36,80
}

\section{Rezension von Robert Hauser und Oliver Parodi, ITAS}

\section{Biographisches Werk und technologisches Kompendium}

Das Buch zur Würdigung des Werkes von Gerhard Banse soll Bestandteil eines Dialogs sein - so die Herausgeber -, eine Sammlung technikwissenschaftlicher und philosophischer Monologe als Antwort auf das Wirken des Jubilars. ${ }^{1}$ Dementsprechend lässt sich die Festschrift in zwei Richtungen lesen: als biographisches Werk über sein Wirken, ergänzt um einige hochkarätige wissenschaftliche Beiträge, oder aber als technologisches Kompendium, strukturiert durch die wissenschaffenden Begegnungen eines Philosophen. ${ }^{2}$

Es zeichnet sich durch eine Fülle und Vielfalt der Themen aus. In 25 Beiträgen werden von 30 Autoren aus neun Ländern und zahlreichen Disziplinen unterschiedliche Reflexionen über Technik geboten. So ist es auch eben jene
Vielfalt, die dem Buch seinen Charakter verleiht, die im selben Zuge aber eine theoretisch vertiefte Rezension des Werkes in der hier gebotenen Kürze unterbindet. Hier lässt sich nur soviel sagen: Als Festschrift und Würdigung des Werkes von Gerhard Banse ist dieses Buch zweifellos gelungen, lässt es doch Tiefe und Breite seines Wirkens - auch als "Wissensnetzwerker", der unermüdlich Fäden spinnt und Knoten knüpft - anschaulich werden.

Als technologisches Fachbuch kann dieses Werk in dreierlei Lesart (erkenntnis-)gewinnbringend sein. Erstens bietet es für Spezialisten wertvolle Artikel $\mathrm{zu}$ bestimmten technologischen oder technik-affinen Themen (s. u.). Zweitens reicht das Kompendium dem breiter technologieinteressierten Leser eine Momentaufnahme aktueller Technikreflexionen, einen Überblick darüber, was im Denken und Forschen über Technik gerade thematisiert wird, en vogue ist und in der Kritik steht. Drittens lässt sich das von Banse geschaffene „Wissensnetzwerk" - zumindest geographisch - klar zwischen Rhein, Alpen, Ostsee und Ural verorten. Damit überspannt es aber auch kulturelle (und hier von Bedeutung: wissenschaftskulturelle) Grenzen in und zwischen Mittelund Osteuropa. Die am Werk beteiligten Autoren wurzeln in unterschiedlichen geistesgeschichtlichen Traditionen; dementsprechend schlagen sich auch ihre kulturellen Unterschiede im Denken über Technik nieder. Damit erlangt dieses Kompendium über die Verbindung unterschiedlicher Denkräume einen besonderen Wert und eignet sich als Einführung in die je andere Wissens-, Wert- und Denkkultur.

\section{Inhaltliche Schwerpunkte}

Im Folgenden wird nun eine Übersicht der angeschnittenen Themenfelder gegeben sowie die Beiträge der einzelnen Autoren darin verortet. Im Zentrum der Festschrift steht klar „Technik“. Zu verstehen ist Technik als Phänomen, das anstößt und Ambivalenz hervorruft, das zur Reflexion zwingt und darüber immer wieder zum Begriff, zum Konzept wird. So sind der theoretischen Auseinandersetzung mit „Technik“ auch üppige 350 der insgesamt 420 Seiten umfassenden Schrift gewidmet. Zum Kompendium wird dieses Werk auch dadurch, dass viele Fragestellungen von den 
Autoren bereits an anderem Ort - und dort ausführlicher - behandelt wurden. Reiz und Gewinn resultieren hier also weniger aus dem Neuen, als vielmehr aus der Verdichtung und Zusammenstellung der Inhalte.

Der Themenvielfalt um Technik verleihen die Kapitel „Konzeptualisierungen der Technikwissenschaften“ (Kap. 1), „Herausforderungen der digitalen Welt" (Kap. 2) sowie „Risiko und Utopie“ (Kap. 3) eine lose Struktur, die gleichzeitig Schwerpunkte des Wirkens von Gerhard Banse markieren. ${ }^{3}$ Das letzte, sehr viel kürzere Kapitel „Begegnungen, Begebenheiten und Erlebnisse“ (Kap. 4) rundet die Festschrift mit persönlichen Begebenheiten aus dessen Leben ab und vermittelt dabei einen Eindruck von der Entstehungsgeschichte des „Banse-Rhizoms“ - ein philosophischer Begriff, der in der poststrukturalistischen Lesart netzwerkartige Formen der Wissensorganisation beschreibt und dazu u.a. Wurzelgeflechte als nichthierarchische Ordnungstrukturen heranzieht. ${ }^{4}$ Unbeteiligte mag hier die Privatheit der Äußerungen befremden. Dem Leser bleibt, sich - je nach Gusto - daran zu erfreuen oder das Kapitel zu überlesen.

Auch in einigen Beiträgen der theoretischen Kapitel werden als Randnotiz Bezüge zur Person des Jubilars angeführt. Dies wirkt für den wissenschaftlich Interessierten zunächst irritierend, durchbricht aber - unseres Erachtens hier wohltuend - die oftmals allzu glatte, rationalistische Oberfläche wissenschaftlicher Lektüre und führt dem Leser - wenn auch hier wohl nicht intendiert - vor Augen, dass sich hinter jeder wissenschaftlichen Wahrheit auch Personen, Dialog und lebensweltliche Beziehungen verbergen.

Die Beiträge im ersten Kapitel kreisen im Wesentlichen um Technik-Konzeptionen im Spannungsfeld von „Wissen“, „Werten“ und „Handeln“. Es werden zum einen verschiedene Ansätze und Positionen hinsichtlich der Beziehung von Technik und Gesellschaft (insb. in der Technikentwicklung) kritisch vorgestellt und verglichen (H. Holz, G. Ropohl, E. Reher). L. Tondl beleuchtet dabei „Wissen“" und „Handeln" im Kontext rationaler Tätigkeit. Ein weiterer Schwerpunkt dieses Kapitels widmet sich dem Thema „Ausbildung“. Machen sich O. Czech / B. Meier letztlich für eine kulturelle Komponente stark, so erläutert $\mathrm{H}$. Wolfgramm den didaktischen Nutzen einer funktionalen Untergliederung von technischen Systemen und Verfahren. Beide Beiträge heben indes auf technische Allgemeinbildung ab. Kapitelschließend rückt aus einer eher Methoden vergleichenden Sicht der Unterschied zwischen Wissens- und Handlungskonzepten der ehemaligen UdSSR, der DDR und der BRD in den Blick (V. Gorokhov).

Das zweite Kapitel beschäftigt sich mit den neuen Herausforderungen der digitalen Welt. Hierbei liegt der Fokus stark auf den Kategorien „Werten“ und „Handeln“ und damit auf ethischen Betrachtungen. Die Problematik der Handlungs- und Nutzungskompetenz in Bezug auf neue Medien (A. Kiepas, G. Zecha) werden dabei ebenso angeschnitten, wie die übergreifenden Themen Globalisierung der Medien, Identitätswandel, veränderte Kommunikationsformen und weitere damit verbundene Auswirkungen auf Kultur (U. Zydek-Bednarczuk). Auch für demokratische Nationalstaaten ergeben sich aus diesen Entwicklungen vielfältige und weit reichende Konsequenzen. Betont werden hier Verwerfungen im Verständnis und Umgang mit Urheberrechten. Sie berühren nicht nur ökonomische Kontexte sondern auch die politischen Grundlagen moderner Demokratien, wie A. Andoni / L. Ayestarán / U. Ursua am Beispiel Spaniens aufzeigen.

Jede Technik birgt grundsätzlich auch die Möglichkeit ihres Versagens bzw. der Fehlfunktion. Der Risikobegriff und damit verbundene Konzepte sind daher wichtiger Bestandteil einer Technologie. „Risiko“ bildet so auch den einen thematischen Pol des dritten Kapitels. Hier werden verschiedene Dimensionen des Risikobegriffs im Kontext von Technikwissenschaft, Technikentwicklung und Technikfolgenabschätzung verhandelt. Dem Begriff des Risikos werden sodann - in letztlicher Ungeklärtheit der Bezüge - die Begriffe der Utopie und der Vision zur Seite gestellt. Im (visionären?) Klima der Technikeuphorie des 20. Jahrhunderts wurden Risiken kaum thematisiert oder marginalisiert. Erst das Versagen von Großtechniken und großflächige Umweltauswirkungen haben in der Folge zu einer Aufwertung und kritischen Reflexion auch des Risikobegriffs geführt (A. Metzner-Szigeth, H. Hörz). Für diesen Paradigmenwechsel steht auch der von I. Hronszky / A. Fésüs thematisierte Über- 
gang vom Risikobegriff zur Konzeptionalisierung von Unsicherheit. Menschen sind direkt oder indirekt von dem (Nicht-)Funktionieren von Technik betroffen. Je nach Betroffenheit entwickeln sie Ver- oder auch Misstrauen in bestimmte Techniken. Daher ist auch der Vertrauensbegriff in Form eines systematisierten Konzeptes berechtigter und erhellender Bestandteil der Risikodebatte (K. Kornwachs).

Visionen und Utopien werden in der wissenschaftlichen Diskussion einerseits immer wieder als treibende Kraft des technischen Fortschritts bezeichnet, andererseits werden aber auch ihre Ambivalenzen betont. Die systematische Untersuchung von Visionen und deren Bedeutung für Gesellschaften könnten offene Fragestellungen für einen möglichen neuen Forschungsbereich des "Vision-Assessments" sein (A. Grunwald). Der Utopie-Begriff hebt sich z. B. in der Konzeption von Ernst Bloch - von dem der „Vision“ ab. Während „Vision“ heute als ,richtungsweisend“ vorwiegend positiv konnotiert ist, hängt dem Utopie-Begriff etwas Zwiespältig-Traumhaftes an. Mit einem historischen Abriss neuzeitlich deutscher Utopien (S. Wollgast) und einer Bestandsaufnahme zeitgenössischer Visionen und Utopien (H.-J. Petsche) endet der theoretische Teil des Buches. Für Petsche gilt es, zukünftig Visionen und Utopien wieder zu verschränken, um nicht Gefahr $\mathrm{zu}$ laufen, das Leben oder wenigstens unsere Humanität zu verlieren (S. 344 f.).

Damit endet der theoretische Monolog des Buches. Der kontroverse Dialog um Technik aber geht weiter ${ }^{5}$ - bereichert um die Beiträge von Gerhard Banse und von den Autoren dieser Festschrift.

\section{Anmerkungen}

1) Gerhard Banse ist Technikwissenschaftler und Philosoph, zu dessen zentralen Lebensstationen eine Philosophieprofessur an der Akademie der Wissenschaften der DDR, eine Honorarprofessur für Allgemeine Technikwissenschaften an der Brandenburgischen Universität Cottbus, ein Aufenthalt an der Europäischen Akademie Bad Neuenahr und seit 1999 eine Mitarbeit am ITAS gehören.

2) „Technologie“ wird hier verstanden im Sinne von Ropohl als umfassende Wissenschaft von der Technik inkl. der Technikphilosophie (Ro- pohl, 1999: Allgemeine Technologie. München. S. 32 f.).

3) Das Buch enthält eine vollständige Bibliografie von Gerhard Banse, die über 30 Seiten einen genaueren Einblick in das Werk des ,Technologen“ bietet.

4) Zur philosophischen Bedeutung des Begriffs „Rhizom“ jenseits der Botanik siehe Deleuze, G.; Guattari, F., 1977: Rhizom. Berlin: Merve, oder auch: http://de.wikipedia.org/wiki/Rhizom_\%28 Philosophie\%29 aufgerufen am 14.6.07

5) Z. B. im Rahmen der Schrift zum Festkolloquium des 60-jährigen Jubilars: Petsche, H.-J.; Krebs, I.; Meinberg, U., 2007 (im Erscheinen): Zwischen Utopie und Risiko - Technikkonzepte im europäischen Integrationsprozess. Berlin: trafo. 